\title{
Increased energy efficiency spin-torque switching of magnetic tunnel junction devices with a higher order perpendicular magnetic anisotropy
}

Cite as: Appl. Phys. Lett. 114, 012404 (2019); https://doi.org/10.1063/1.5049837

Submitted: 25 July 2018 . Accepted: 15 December 2018. Published Online: 07 January 2019

Marion Lavanant, Sebastien Petit-Watelot, Andrew D. Kent, and Stephane Mangin (D)
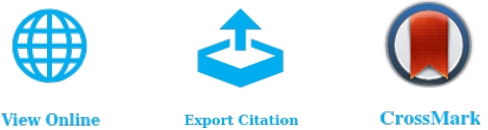

\section{ARTICLES YOU MAY BE INTERESTED IN}

Ultrafast field-free magnetization switching using bi-directional spin Hall current and antiferromagnetic interlayer exchange

Applied Physics Letters 114, 012403 (2019); https://doi.org/10.1063/1.5063423

Interfacial electric field and spin-orbitronic properties of heavy-metal/CoFe bilayers

Applied Physics Letters 114, 012401 (2019); https://doi.org/10.1063/1.5043444

Brownian motion of skyrmion bubbles and its control by voltage applications Applied Physics Letters 114, 012402 (2019); https://doi.org/10.1063/1.5070101
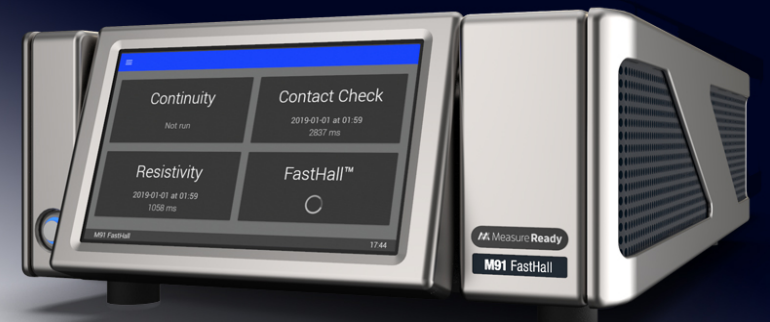

Measure Ready M91 FastHall ${ }^{\text {TM }}$ Controller

A revolutionary new instrument for complete Hall analysis 


\title{
Increased energy efficiency spin-torque switching of magnetic tunnel junction devices with a higher order perpendicular magnetic anisotropy
}

\author{
Cite as: Appl. Phys. Lett. 114, 012404 (2019); doi: 10.1063/1.5049837 \\ Submitted: 25 July 2018 - Accepted: 15 December 2018 • Published Online: \\ 07 January 2019
}

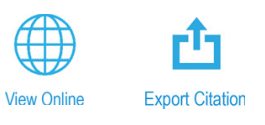

Marion Lavanant, ${ }^{1,2}$ Sebastien Petit-Watelot, ${ }^{1}$ Andrew D. Kent, ${ }^{2}$ and Stephane Mangin ${ }^{1, a)}$ (iD

\author{
AFFILIATIONS \\ ${ }^{7}$ Institut Jean Lamour, Université de Lorraine CNRS UMR 7198, Nancy, France \\ ${ }^{2}$ Center for Quantum Phenomena, Department of Physics, New York University, New York, New York 10003, USA \\ a) Author to whom correspondence should be addressed: stephane.mangin@univ-lorraine.fr
}

\begin{abstract}
We study the influence of a second order magnetic anisotropy on magnetization reversal by spin transfer torque in perpendicularly magnetized magnetic tunnel junctions (pMTJs). Using a macrospin model to describe the dynamics of the free layer, analytical solutions for the switching voltage and the voltage threshold for precession are determined as a function of the first and second order magnetic anisotropies. To compare the spin-transfer-torque energy efficiency to that of a classical pMTJ, a junction without the second order anisotropy term, we compare these cases at a fixed energy barrier to thermally activated reversal. We show that the critical voltage for switching can be reduced by a factor 0.7 when the ratio of the second to the first order magnetic anisotropy is $1 / 3$. Importantly, the switching time can be reduced by nearly a factor of two for this magnetic anisotropy ratio. These results highlight an important and practical method to increase the spin-torque efficiency, while reducing the energy dissipation and switching time in magnetic random access memory devices.
\end{abstract}

Published under license by AIP Publishing. https://doi.org/10.1063/1.5049837

Magnetic tunnel junctions (MTJs) with perpendicular anisotropy have been developed for memory applications such as magnetic random access memory (MRAM) since $2010 .^{1-3}$ In order to optimize these devices, material solutions need to be found to reduce the writing power and the writing duration of information in a memory cell while keeping a sufficient thermal stability. In this article, we focus on the magnetization behavior of the free layer of a perpendicular magnetic tunnel junction (pMTJ) with a second order anisotropy term in the presence of the anti-damping (Slonczewski-like) spin transfer torque (STT). 4

The existence of a second order of magnetocrystalline anisotropy in $\mathrm{MgO} / \mathrm{CoFeB} / \mathrm{Ta}$ structures was shown in 2015 by Shaw et al..$^{10}$ for thin CoFeB layers $(\sim 1 \mathrm{~nm})$ using ferromagnetic resonance spectroscopy (FMR). The same year Sun showed that higher order magnetic anisotropies can arise in thin films when the perpendicularly magnetic anisotropy is concentrated at an interface (e.g., the $\mathrm{CoFeB} / \mathrm{MgO}$ interface) and there is moderate exchange coupling between the interface moments and the rest of the thin layer. ${ }^{11}$ Both references suggested that this higher order anisotropy would increase the switching efficiency of the magnetization-but did not provide an analysis of this effect. Subsequently, several experimental studies focused on either this structure, putting in evidence the second order magnetic anisotropy for pMTJs with thin CoFeB layers (1.2-1.35 nm) using FMR, or magnetoresistance for patterned structures. ${ }^{12-14}$ Other studies explored the influence of this effect on pMTJ state diagrams. ${ }^{15-19}$ Reference 19 considered the influence of a second order anisotropy on the switching current.

In contrast, this article focuses on an analytical description in the macrospin limit of the precession voltage $V_{p}$, the switching voltage $\mathrm{V}_{s w}$, and the corresponding switching duration, which determine the energy required for switching, as a function of the anisotropy ratio for a constant energy barrier. In order to evaluate the effect of the second order term on the magnetization dynamics, we consider the macrospin model to represent this system. ${ }^{6,7}$ This model is solved analytically to extract the critical voltage and the time depend dynamics is numerically calculated from the Landau-Lifshitz-GilbertSlonczewski (LLGS) equation. ${ }^{8,9}$ We conclude that the energy 
efficiency of an MRAM device can be increased by introducing a second order anisotropy.

Introducing a second order magnetic anisotropy term results in the following expression for the energy density:

$$
\mathcal{E}_{\text {ani }}(\theta)=-K_{1} \cos ^{2} \theta-K_{2} \cos ^{4} \theta,
$$

where $K_{1}$ and $K_{2}$ are the first and the second anisotropy constants, respectively, and $\theta$ is the angle with the normal to the sample plane. In this article, $K_{1}$ will be considered positive in order to promote an out-of-plane easy axis and $K_{2}$ negative, as this aids the switching and is what is found experimentally for $\mathrm{CoFeB} / \mathrm{MgO}$ interfaces.

The thermal stability of the device is set by the energy barrier height $E_{b}$ which typically in commercial devices is designed to be $60 k_{\mathrm{B}} \mathrm{T}$ to ensure long term data retention, where $\mathrm{T}$ is the device operating temperature (typically, $300 \mathrm{~K}$ ) and $k_{\mathrm{B}}$ is Boltzmann's constant. The total anisotropy energy $E_{\text {tot }}=\mathcal{E}_{\text {tot }} \mathcal{V}$ is given by the volume of the free layer $\mathcal{V}$ multiplied by the total anisotropy energy density $\mathcal{E}_{\text {tot }}$ which itself is composed of the shape anisotropy and the magnetocrystalline anisotropy energy density. $\mathcal{E}_{\text {tot }}$ is given by

$$
\begin{aligned}
\mathcal{E}_{\text {tot }}(\theta, \phi) & =\frac{1}{2} \mu_{0} M_{\mathrm{s}}^{2} \cos ^{2} \theta-\mathrm{K}_{1} \cos ^{2} \theta-\mathrm{K}_{2} \cos ^{4} \theta \\
& \equiv-K_{\text {eff }} \cos ^{2} \theta-\mathrm{K}_{2} \cos ^{4} \theta=-K_{\text {eff }} \cos ^{2} \theta\left(1-\frac{\left|\mathrm{K}_{2}\right|}{K_{\text {eff }}} \cos ^{2} \theta\right),
\end{aligned}
$$

where $M_{s}$ is the saturation magnetization and there is no applied magnetic field. We define the effective anisotropy coefficient to be $K_{\text {eff }}=K_{1}-\frac{1}{2} \mu_{0} \mathrm{M}_{\mathrm{s}}{ }^{2}$ which is composed of the first order of magnetocrystalline anisotropy and the shape anisotropy. In the absence of a second-order anisotropy term, an out-of-plane uniaxial anisotropy is favored for $K_{\text {eff }}>0$. When $K_{2}$ is introduced, two cases can be distinguished: $K_{2}<\frac{K_{\text {eff }}}{2}$, the system has out-of-plane uniaxial anisotropy. $K_{2}>\frac{K_{\text {eff }}}{2}$, an easy cone state is favored. In the presence of the cone state, the position of the equilibrium angle $\theta_{\text {cone }}$ is given by $\cos \theta_{\text {cone }}=\sqrt{\frac{K_{e f f}}{2\left|K_{2}\right|}}$. The energy density barrier $\mathcal{E}_{b}$ depends on the presence or absence of the cone state, and we obtain the following relation:

$$
\left\{\begin{array}{l}
\mathcal{E}_{b}=K_{\text {eff }}+K_{2} \text { for } \quad\left|K_{2}\right| \leq \frac{K_{e f f}}{2} \\
\mathcal{E}_{b}=\frac{K_{e f f}{ }^{2}}{4\left|K_{2}\right|} \text { for } \quad\left|K_{2}\right|>\frac{K_{e f f}}{2} .
\end{array}\right.
$$

In order to evaluate the influence of $K_{2}$ on the switching voltage $\mathrm{V}_{s w}$, we fix $\mathcal{E}_{b}$ and $M_{s}$ as the ratio $\frac{\left|K_{2}\right|}{K_{\text {eff }}}$ varies. Figure 1(a) presents the evolution of the energy density landscape normalized by the energy barrier as a function of $\theta$ for different values of $\frac{\left|K_{2}\right|}{K_{\text {eff }}}$. We limit our study to $\frac{\left|K_{2}\right|}{K_{\text {eff }}} \leq 1$ where $\theta_{\text {cone }} \in\left[0, \frac{\pi}{4}\right]$. The reference energy density landscape without second order magnetic anisotropy is shown in black. An increase in $\frac{\left|K_{2}\right|}{K_{e f f}}$ corresponds to a widening of the potential well, and for $\frac{\left|K_{2}\right|}{K_{\text {eff }}}=1 / 2$, we observe, as
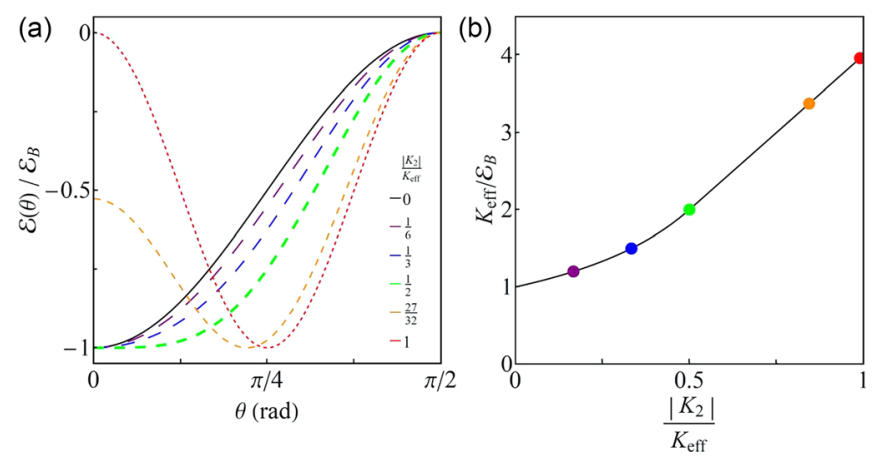

FIG. 1. (a) Normalized energy density landscape as a function of the free layer magnetization position for a pMTJ with a constant energy barrier for different ratios of second order anisotropy $\boldsymbol{K}_{2}$ and effective anisotropy $\boldsymbol{K}_{\text {eff }}=\boldsymbol{K}_{1}-\frac{1}{2} \boldsymbol{\mu}_{0} \boldsymbol{M}_{\boldsymbol{s}}{ }^{2}$ coefficients. (b) Normalized effective anisotropy coefficient as a function of $\frac{\left|\boldsymbol{K}_{2}\right|}{\boldsymbol{K}_{\text {eff }}}$

expected, the appearance of an easy cone state and a shift of the position of the equilibrium angle toward the in-plane direction. For a constant $K_{\text {eff }}$, the addition of (a negative) $K_{2}$ would decrease the height of the energy barrier; however, we want to focus on the effect of a ratio $\frac{\left|K_{2}\right|}{K_{\text {eff }}}$ on the switching voltage efficiency in which the energy barrier $\mathcal{E}_{b}$ is fixed, as shown in Fig. 1(b).

We are now interested in finding the switching voltage for different ratios of $\frac{\left|K_{2}\right|}{K_{\text {eff }}}$, while keeping a constant energy barrier $\mathcal{E}_{b}$. The aim is to characterize the magnetization dynamics as a function of the applied voltage. The two effects of the spin torque expected in this geometry are the precession and the switching of the magnetization. We consider the LLGS equation written as

$$
\frac{d \boldsymbol{m}}{d t}=-\gamma_{0} \boldsymbol{m} \times \boldsymbol{H}_{\text {eff }}-\alpha \boldsymbol{m} \times \frac{d \boldsymbol{m}}{d t}+\gamma_{0} a_{\mathrm{V}} \mathrm{V} \boldsymbol{m} \times(\boldsymbol{m} \times \boldsymbol{p}),
$$

where the first right hand side term is the conservative term, depending on the effective field, and the two other terms are the non-conservative torques, the damping torque $(\alpha \ll 1)$ and the Slonczewski spin transfer torque as discussed theoretically in Ref. 20, where it is shown that the spin-transfer torque is directly proportional to the applied bias voltage, as observed experimentally in Ref. 21.

We will take $\boldsymbol{p}$ to be normal to the film plane, that is, we consider the fixed layer to be perpendicularly magnetized. The process followed to develop our computation was to express the LLGS equation in spherical coordinates $\left(\boldsymbol{e}_{\rho}, \boldsymbol{e}_{\theta}, \boldsymbol{e}_{\phi}\right)$ and then project Eq. (3) onto $\boldsymbol{e}_{\theta}$ and $\boldsymbol{e}_{\phi}$. The domain of the definition of the angle solution is $\theta \in\left[0, \frac{\pi}{2}\right]$, and the solution is symmetric for the lower hemisphere. From Eq. (1), we get $\boldsymbol{H}_{\text {eff }}$ $=\frac{2}{\mu_{0} \mathrm{M}_{\mathrm{s}}}\left(\mathrm{K}_{\text {eff }}+2 \mathrm{~K}_{2} \cos ^{2} \theta\right) \cos \theta \sin \theta \boldsymbol{e}_{\theta}$, and the LLGS equation can be written as

$$
\left\{\begin{array}{l}
\dot{\theta}=\left(\gamma_{0} a_{\mathrm{V}} \mathrm{V}-\alpha \dot{\phi}\right) \sin \theta \\
\dot{\phi} \sin \theta=\frac{2 \gamma_{0}}{\mu_{0} \mathrm{M}_{\mathrm{s}}}\left(\mathrm{K}_{\text {eff }}+2 \mathrm{~K}_{2} \cos ^{2} \theta\right) \cos \theta \sin \theta+\alpha \dot{\theta} .
\end{array}\right.
$$


It has a trivial solution with $\theta=0$ that in turn gives $\dot{\theta}=0$ and indicates that there is no motion possible for a magnetization that remains precisely out of the plane. If we allow $\theta$ to be different from zero, the equations can be divided by $\sin \theta$, but the resulting equations cannot be solved without numerical integration. However, due to the cylindrical symmetry of the system, if the magnetization is driven in a precessional state, $\dot{\theta}=0$, and the precession frequency is given by $\dot{\phi}$. In that case, Eq. (4) can be rearranged as

$$
\left\{\begin{array}{l}
\dot{\phi}=\frac{\gamma_{0} a_{\mathrm{V}}}{\alpha} \mathrm{V} \\
\dot{\phi}=\frac{2 \gamma_{0}}{\mu_{0} \mathrm{M}_{\mathrm{s}}}\left(\mathrm{K}_{\text {eff }}+2 \mathrm{~K}_{2} \cos ^{2} \theta\right) \cos \theta .
\end{array}\right.
$$

The first equation [Eq. 5(a)] shows that the magnetization precession frequency is a linearly increasing monotonic function of the voltage and the second equation [Eq. 5(b)] implies that the precession frequency is bounded by $\frac{2 \gamma_{0}}{\mu_{0} M_{s}}\left(K_{e f f}+2 K_{2} \cos ^{2} \theta\right) \cos \theta$. Combining the above two equations, we can get the evolution of the voltage with respect to the dynamic equilibrium polar angle $\theta_{d y n}$. As Eq. 5(b) is bounded, we can define two limit voltages $V_{1}$ and $V_{2}$ where the solution of the LLGS equation changes. These values are directly linked to the $\theta_{d y n}$ bounding values. The lower bound of $\theta_{d y n}$ is given by the equilibrium angle, and thus, $\theta_{d y n_{1}}=0$ for $\frac{\left|K_{2}\right|}{K_{\text {eff }}} \leq \frac{1}{2}$ and $\cos \theta_{\text {dyn }}=\cos \theta_{\text {cone }}=\sqrt{\frac{K_{\text {eff }}}{2\left|K_{2}\right|}}$ for $\frac{\left|K_{2}\right|}{K_{\text {eff }}} \geq \frac{1}{2}$. Solving the system of equations for the upper bound gives $\cos \theta_{d y n_{1}}=\sqrt{\frac{K_{\text {eff }}}{6\left|K_{2}\right|}}$. Consequently, $V_{1}$ and $V_{2}$, denoting regime transitions and expressed as functions of the magnetic anisotropy, are given by

$$
\mathrm{V}_{1}=\left\{\begin{array}{lll}
\frac{\alpha}{a_{\mathrm{V}}} \frac{2}{\mu_{0} M_{\mathrm{s}}}\left(\mathrm{K}_{\text {eff }}+2 \mathrm{~K}_{2}\right) & \text { for } & \frac{\left|\mathrm{K}_{2}\right|}{\mathrm{K}_{\text {eff }}} \leq \frac{1}{2} \\
0 & \text { for } & \frac{\left|\mathrm{K}_{2}\right|}{\mathrm{K}_{\text {eff }}} \geq \frac{1}{2}
\end{array}\right.
$$

and

$$
\mathrm{V}_{2}=\frac{4}{3} \frac{\alpha}{a_{\mathrm{V}}} \frac{\mathrm{K}_{\text {eff }}}{\mu_{0} \mathrm{M}_{\mathrm{s}}} \sqrt{\frac{\mathrm{K}_{\text {eff }}}{6\left|\mathrm{~K}_{2}\right|}} .
$$

Both voltages are presented as a function of $\frac{\left|K_{2}\right|}{K_{\text {eff }}}$ in Fig. 2(a) keeping a constant energy barrier $\varepsilon_{b}$ and in Fig. 2(b) the magnetization trajectory is shown for each area in the diagram using a direct numerical integration of the LLGS equation. ${ }^{8}$

As $V_{1}$ and $V_{2}$ have a bifurcation point for $\frac{\left|K_{2}\right|}{K_{\text {eff }}}=\frac{1}{6}$, they define a four-area diagram, each noted by a roman numeral. By solving numerically the LLGS equation, we identify the archetypal trajectories of each area. When the magnetization is in the first (I) or the second (II) area, it switches directly to the opposite hemisphere; this corresponds to the area between $V_{1}$ and $V_{2}$ before the bifurcation and for voltages larger than $V_{2}$. In the area below $\mathrm{V}_{1}$ (III), the stable state is the out-of-plane equilibrium position. For the fourth area (IV), i.e., between $V_{1}$ and $V_{2}$ and $\frac{\left|K_{2}\right|}{K_{\text {eff }}}>\frac{1}{6}$, we find an area with a steady state precession, which exists independently of the cone state. From this diagram, we can define

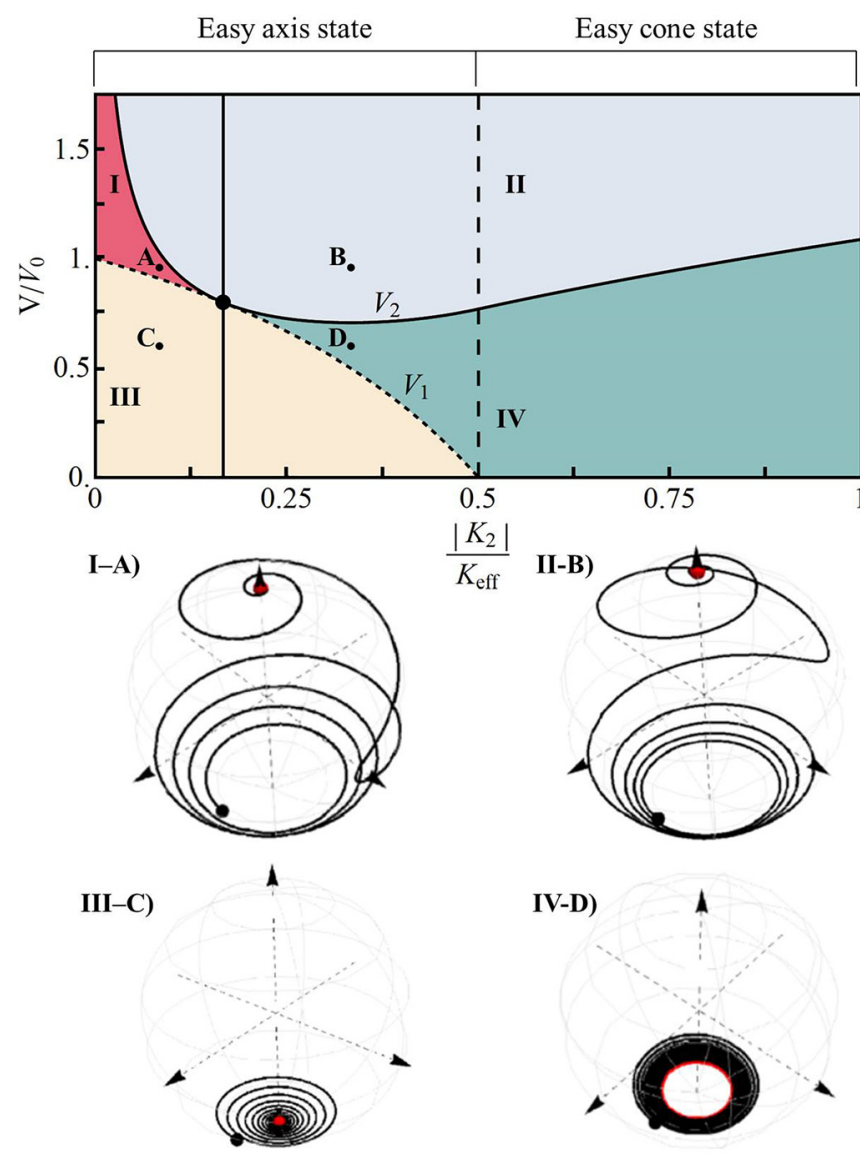

FIG. 2. Solutions of LLGS for the precession and the switching of magnetization as a function of voltage. $\boldsymbol{V}_{1}$ (dashed line) and $\boldsymbol{V}_{2}$ (thick line) as a function of $\left|\boldsymbol{K}_{2}\right| / \boldsymbol{K}_{\text {eff }}$ for a constant energy barrier, with the large black dot denoting the case when the two voltages are equal. The red area (I) corresponds to a switching area between $\boldsymbol{V}_{1}$ and $\boldsymbol{V}_{2}$, and the grey area (II) to the switching area above both $\boldsymbol{V}_{1}$ and $\boldsymbol{V}_{2}$. In the yellow area (III), the magnetization does not switch, and in the green area (IV), the magnetization presents a precessional behavior. Below, typical magnetization trajectories in the four areas are given I and II: magnetization switching, III: non-switching, IV: precession. The following values have been used: $a_{V}=6.37$ $\times 10^{3} \mathrm{~A} \mathrm{~m}^{-1} V^{-1}$ and $\alpha=0.01$.

the switching voltage $V_{s w}$ as $V_{1}$ for $\frac{\left|K_{2}\right|}{K_{e f f}}<\frac{1}{6}$ and $V_{2}$ for $\frac{\left|K_{2}\right|}{K_{\text {eff }}} \geq \frac{1}{6}$ and the precession voltage $V_{p}$ is $V_{1}$ for $\frac{\left|K_{2}\right|}{K_{\text {eff }}} \geq \frac{1}{6}$.

An important result of this analysis is that the ratio $\left|K_{2}\right| / K_{\text {eff }}=13$ minimizes the switching voltage compared to the switching voltage $V_{0}$ of a pMTJ with no second order anisotropy but with the same energy barrier [Fig. 3(a)]. Even when the cone state is not present, the steady state precession induces a motion of the magnetization before switching, increasing the efficiency of the magnetization reversal for the same energy barrier. A pMTJ with this ratio in anisotropy would have a decreased switching voltage with little reduction of its magnetoresistance.

We also investigate the duration of the switching process under STT by integrating numerically the LLGS equation in 


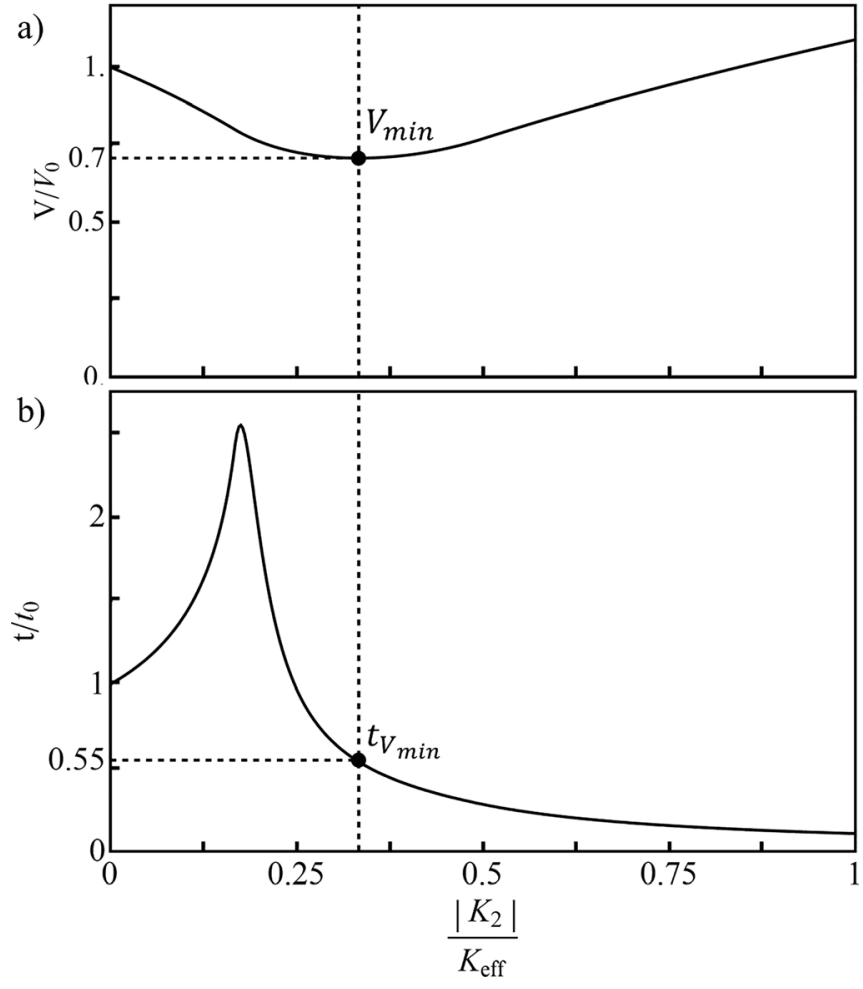

FIG. 3. (a) Switching voltage and (b) switching time as a function of $\frac{\left|K_{2}\right|}{K_{0}}$ normalized, respectively, by the Switching voltage $V_{0}$ and the switching time $\boldsymbol{t}_{0}$ off a reference pMTJ with no second order anisotropy.

pMTJs for various ratios $\frac{\left|K_{2}\right|}{K_{e f f}}$. The initial angle of the magnetization is tilted 0.05 rad away from the normal axis, and the switching time is identified when the magnetization changes hemisphere, i.e., when it overcomes the energy barrier.

Figure 3 shows the switching voltage (a) and the switching time (b) in pMTJs with different values of $\frac{\left|K_{2}\right|}{K_{\text {eff }}}$ and for a voltage overdrive of $1 \%$ for Fig. 3(b). From Fig. 3(b), two switching regimes are identified, below and above the bifurcation point $\left(\frac{\left|K_{2}\right|}{K_{\text {eff }}}=\frac{1}{6}\right)$. The peak in the switching time is associated with the coexistence of both magnetization precession and switching at this point.

Both parameters are normalized with the switching voltage $\mathrm{V}_{0}$ and switching time $t_{0}$ of the reference pMTJ with no second order anisotropy. Another important feature is that for $\frac{\left|K_{2}\right|}{K_{\text {eff }}}=\frac{1}{3}$, where the switching voltage is minimum, reduced by $\frac{V_{\text {min }}}{V_{0}}=0.71$, the switching time is also reduced by $\frac{t_{v_{\text {min }}}}{t_{0}}=0.55$. A pMTJ with this ratio in anisotropy at a constant energy barrier would see not only its switching voltage having decreased but also its switching time, while presenting no reduction of the tunnel magnetoresistance (TMR) ratio, since the system remains for this value of $\frac{\left|K_{2}\right|}{K_{e f f}}$, and for sufficiently low reading voltage in a full pMTJ geometry. The Joule energy required for switching is a product of the power supplied and the time. So, the reduction in both the switching voltage and the switching time leads to a more energy efficient spin-torque device.

In conclusion, the magnetization switching and precession in pMTJs in the presence of a second order anisotropy $K_{2}$ have been studied while keeping a constant energy barrier (constant thermal stability). From the LLGS equation in the macrospin approximation, we find analytical solutions of the boundaries for several different regimes as a function of $\frac{\left|K_{2}\right|}{K_{e f f}}$. The numerical integration of the LLGS equation allowed identifying these different regimes: a switching regime, a non-switching regime, and a precession regime, the latter appearing before the creation of the cone state in the energy landscape. From the equations of the switching voltage and the precession voltage, a bifurcation is found for $\frac{\left|K_{2}\right|}{K_{\text {eff }}}=\frac{1}{6}$, which corresponds to an increase in switching time. The switching duration decreases with increasing voltage beyond this particular ratio $\frac{\left|K_{2}\right|}{K_{\text {efft }} \mid}=\frac{1}{6}$. Moreover, we could demonstrate that for a well chosen $\frac{K_{2} \mid}{K_{\text {eff }}}$ ratio, the switching voltage is reduced by $29 \%$ and the switching time by $45 \%$ while the energy barrier is kept constant. These findings could have major consequences on the materials used for the next generation of STTMRAM. We note that a more detailed analysis of the thermal stability would include how the prefactor in the rate of thermally activated transitions varies with $\mathrm{K}_{2}$. Further, a 3D micromagnetic calculation should be carried out to examine the limits to the macrospin approximation used in our study. ${ }^{22,23}$

The research at NYU was supported by Grant No. NSFDMR-1610416. The research at Institut Jean Lamour was supported by the French PIA project "Lorraine Université d'Excellence," reference ANR-15-IDEX-04-LUE. Marion Lavanant's Phd was supported by a Cifre grant from Vinci Technologie.

\section{REFERENCES}

${ }^{7}$ S. Ikeda, K. Miura, H. Yamamoto, K. Mizunuma, H. D. Gan, M. Endo, S. Kanai, J. Hayakawa, F. Matsukura, and H. Ohno, "A perpendicularanisotropy CoFeB MgO magnetic tunnel junction," Nat. Mater. 9(2804), 721-724 (2010).

${ }^{2}$ D. C. Worledge, G. Hu, D. W. Abraham, J. Z. Sun, P. L. Trouilloud, J. Nowak, S. Brown, M. C. Gaidis, E. J. O'Sullivan, and R. P. Robertazzi, "Spin torque switching of perpendicular $\mathrm{Ta}|\mathrm{CoFeB}| \mathrm{MgO}$ based magnetic tunnel junctions," Appl, Phys. Lett. 98, 022501 (2011).

${ }^{3}$ D. C. Worledge, G. Hu, D. W. Abraham, P. L. Trouilloud, and S. Brown, "Development of perpendicularly magnetized $\mathrm{Ta}|\mathrm{CoFeB}|$ mgo-based tunnel junctions at IBM," J. Appl. Phys. 115, 172601 (2014).

${ }^{4}$ J. C. Slonczewski, "Current-driven excitation of magnetic multilayers," J. Magn. Magn. Mater. 159(1-2), L1-L7 (1996).

${ }^{5}$ L. Berger, "Emission of spin waves by a magnetic multilayer traversed by a current," Phys. Rev. B 54(13), 9353 (1996).

${ }^{6}$ E. C. Stoner and E. P. Wohlfarth, "A mechanism of magnetic hysteresis in heterogeneous alloys," Philos. Trans. R. Soc. A 240(826), 599-642 (1948).

${ }^{7}$ J. Cucchiara, E. E. Fullerton, A. D. Kent, J. Z. Sun, Y. Henry, and S. Mangin, "Current-induced magnetization reversal in terms of power dissipation," Phys. Rev. B 84, 100405(R) (2011).

${ }^{8}$ G. Consolo, G. Gubbiotti, L. Giovannini, and R. Zivieri, "Lagrangian formulation of the linear autonomous magnetization dynamics in spin-torque auto-oscillators," Appl. Math. Comput. 217(8204), 8204 (2011).

${ }^{9}$ M. Lavanant, S. Petit-Watelot, A. D. Kent, and S. Mangin, "State diagram of a perpendicular magnetic tunnel junction driven by spin transfer torque: A power dissipation approach," J. Magn. Magn. Mater. 428, 293-299 (2017). 
${ }^{10}$ J. M. Shaw, H. T. Nembach, M. Weiler, T. J. Silva, M. Schoen, J. Z. Sun, and D. C. Worledge, "Perpendicular magnetic anisotropy and easy cone state in Ta/CoFeB/MgO," IEEE Magn. Lett. 6, 3500404 (2015).

${ }^{11}$ J. Z. Sun, "Consequences of an interface-concentrated perpendicular magnetic anisotropy in ultrathin $\mathrm{CoFeB}$ films used in magnetic tunnel junctions," Phys. Rev. B 91, 174429 (2015).

${ }^{12}$ Y. Fu, I. Barsukov, J. Li, A. M. Goncalves, C. C. Kuo, M. Farle, and I. N. Krivorotov, "Temperature dependence of perpendicular magnetic anisotropy in CoFeB thin films," Appl. Phys. Lett. 108, 142403 (2016)

${ }^{13}$ P. H. Jang, S. W. Lee, and K. J. Lee, "Spin-transfer-torque-induced zerofield microwave oscillator using a magnetic easy cone state," Curr. Appl. Phys. 16(12), 1550-1553 (2016).

${ }^{14}$ A. Timopheev, R. Sousa, M. Chshiev, T. Nguyen, and B. Dieny, "Second order anisotropy contribution in perpendicular magnetic tunnel junctions," Sci. Rep. 6, 26877 (2016).

${ }^{15}$ N. Strelkov, A. Timopheev, R. C. Sousa, M. Chshiev, L. D. BudaPrejbeanu, and B. Dieny, "Stability phase diagram of a perpendicular magnetic tunnel junction in noncollinear geometry," Phys. Rev. B 95, 184409 (2017).

${ }^{16}$ A. A. Timopheev, B. M. S. Teixeira, R. C. Sousa, S. Aufret, T. N. Nguyen, L. D. Buda-Prejbeanu, M. Chshiev, N. A. Sobolev, and B. Dieny, "Inhomogeneous free layer in perpendicular magnetic tunnel junctions and its impact on the effective anisotropies and spin transfer torque switching efficiency," Phys. Rev. B 96, 014412 (2017).
${ }^{17}$ S. Perna, R. Tomasello, T. Scimone, M. d'Aquino, C. Serpico, M. Carpentieri, and G. Finocchio, "Influence of the second-order uniaxial anisotropy on the dynamical proprieties of magnetic tunnel junctions," IEEE Trans. Magn. 53(4), 1400107 (2017)

${ }^{18}$ S. J. Yun, K.-J. Lee, and S. H. Lim, "Critical switching current density induced by spin Hall effect in magnetic structures with first and second-order perpendicular magnetic anisotropy," Nat. Sci. Rep. 7, 15314 (2017).

${ }^{19}$ R. Matsumoto, H. Arai, S. Yuasa, and H. Imamura, "Efficiency of spintransfer-torque switching and thermal-stability factor in a spin-valve nanopillar with first- and second-order uniaxial magnetic anisotropies," Phys. Rev. Appl. 7, 044005 (2017).

${ }^{20} \mathrm{~J}$. C. Slonczewski, "Currents, torques, and polarization factors in magnetic tunnel junctions," Phys. Rev. B 71, 024411 (2005).

${ }^{21}$ S. Petit, N. de Mestier, C. Baraduc, C. Thirion, Y. Liu, M. Li, P. Wang, and B. Dieny, "Influence of spin-transfer torque on thermally activated ferromagnetic resonance excitations in magnetic tunnel junctions," Phys. Rev. B 78, 184420 (2008).

${ }^{22}$ D. P. Bernstein, B. Brauer, R. Kukreja, J. Stohr, T. Hauet, J. Cucchiara, S. Mangin, J. A. Katine, T. Tyliszczak, K. W. Chou, and Y. Acremann, Phys. Rev. B 83, 180410 (2011).

${ }^{23}$ P. Bouquin, S. Rao, G. S. Kar, and T. Devolder, "Size dependence of spintorque switching in perpendicular magnetic tunnel junctions," Appl. Phys. Lett. 113, 222408 (2018). 